DO-TH 06/06

July 26, 2006

\title{
Neutrino Phenomenology, Dark Energy and Leptogenesis from pseudo-Nambu-Goldstone Bosons
}

\author{
C.T. Hill ${ }^{1}$, I. Mocioiu ${ }^{2}$, E.A. Paschos ${ }^{3}$ and U. Sarkar ${ }^{3,4}$ \\ 1 Fermi National Laboratory, P.O. Box 500, Batavia, Illinois 60510, USA \\ 2 Pennsylvania State University, 104 Davey Lab, University Park, PA 16802-6300, USA \\ 3 Institut für Physik, Universität Dortmund, D-44221 Dortmund, Germany \\ 4 Physical Research Laboratory, Ahmedabad 380009, India
}

\begin{abstract}
We consider a model of dynamical neutrino masses via the see-saw mechanism. Nambu-Goldstone bosons (majorons) arise associated with the formation of the heavy right-handed majorana masses. These bosons then acquire naturally soft masses (become pNGB's) at loop level via the Higgs-Yukawa mass terms. These models, like the original neutrino pNGB quintessence schemes of the 1980's [1, 2] that proceed through the Dirac masses, are natural, have cosmological implications through mass varying neutrinos, long range forces, and provide a soft potential for dark energy. We further argue that these models can explain leptogenesis naturally through the decays of the right-handed neutrinos.
\end{abstract}




\section{Introduction}

The conventional explanation for neutrino mass is the see-saw mechanism [3]. In this scheme, sterile right-handed neutrinos have very large (grand unification scale) majorana masses $M_{i}$. The standard model is then a low energy effective theory, obtained by integrating out the right-handed neutrinos, leading to suppressed induced Majorana masses for the lefthanded neutrinos, of order $v^{2} / M$, where $v$ is the electroweak scale $\mathrm{VEV}, v=175 \mathrm{GeV}$ or the typical Dirac mass for leptons. The large $M_{i}$ break the global lepton numbers of the right-handed neutrinos. It is natural to hypothesize that this symmetry breaking is dynamical, much like the chiral dynamics of QCD, and is quite similar to the Cooper pairing in a superconductor. The spontaneous breaking of these global symmetries will produce massless Nambu-Goldstone bosons (NGB's). In the present case such NGB's associated with majorana mass generation are termed "majorons" [4]. It is possible that a whole category of new particles, NGB's such as axions [5], majorons, familons, etc., exists in nature, reflecting the generic breaking of multitudinous global symmetries.

The spontaneously broken global symmetries of the right-handed neutrinos will generally be explicitly broken as well. This explicit breaking must, at least, arise in the Yukawa couplings of the right-handed and left-handed neutrinos to the Higgs field of the standard model, i.e., in the Dirac mass terms that marry the left-handed $I=1 / 2$ and right-handed sterile neutrinos. In the present paper we will assume that this is the primary source of the explicit global symmetry breaking, and that any other sources of such breaking do not lead to significantly larger effects than these. It then follows that the majorons will become pseudoNambu-Goldstone bosons (pNGB's) at least via loop diagrams that involve insertions of the various Yukawa vertices in the presence of the Higgs vacuum expectation value (VEV). These loops require sufficiently many insertions that they become finite, and the pNGB masses are then "calculable," in a sense discussed by Georgi and Pais [6]. This was further developed by Hill and Ross [7] to construct technically natural models, involving CP-violation and other symmetry breaking effects, that give rise to calculable novel long range forces in the context 
of the Standard Model. The finiteness of the induced pNGB masses is analogous to what happens in deconstructed extra dimensions [8]. E.g., for QED in $D=5$ an NGB arises that is $\int d x^{5} A_{5}$, the Wilson line of the fifth component of the photon vector potential integrated over the compact fifth dimension. In deconstruction, this is finite when $N \geq 3$ lattice slices are taken for the fifth dimension. Extra dimensions or deconstruction offer a method of solving the naturalness problems associated with large decay constants of axions and other pNGB's [9].

In the case of majoronic pNGB's the mass scales of majorons are generally small and majorons will have large finite Compton wavelengths. Neutrino majorons can thus have astrophysical/cosmological implications. This was first recognized many years ago as a general mechanism to induce large distance or late-time effects in the not-so early universe by Hill, Schramm and Fry [1], and was subsequently developed in greater detail by Frieman, Stebbins, Waga, Kolb et.al. [2]. These models, which exploit spontaneous chiral symmetry breaking associated with Dirac masses, have the virtue of naturalness, and also, unlike dilatonic schemes and a host of random scalar potential models, we have prior experience in physics with pNGB's (eg., the pion is a pNGB). These models are amongst the original, and are well motivated as they are the first natural, quintessence models [1, 2, 10]. They are also the original models of mass varying neutrinos ("MAVAN's"). This general phenomenon has been largely rediscovered in recent years [11, 12, 13], and the original models can provide a natural origin to an "acceleron" field. The present analysis incorporates many features of [1, 2] within the context of the seesaw mechanism and the dynamical generation of right-handed majorana masses.

We further investigate the possibility of a majoronic pNGB as an "acceleron," studying its implications for the leptonic sector, i.e., leptogenesis. 


\section{Two Generation pNGB Model}

Consider an extension of the standard model with two right-handed neutrinos $N_{1}$ and $N_{2}$, which are singlets under the standard model gauge groups. There are no majorana masses for these fields and hence lepton number is not explicitly broken at this level. We introduce two singlet scalar fields, $\Phi_{1}(x)$ and $\Phi_{2}(x)$, which are also singlets under the standard model gauge groups. We postulate that these fields couple with the right-handed neutrinos as:

$$
\mathcal{L}_{M}=\frac{1}{2} \alpha_{1} \bar{N}_{1} N_{1}^{c} \Phi_{1}+\frac{1}{2} \alpha_{2} \bar{N}_{2} N_{2}^{c} \Phi_{2}
$$

Then these scalars will acquire vacuum expectation values (VEV's) and will give Majorana masses to the right-handed neutrinos. The model possesses a global $U(1)_{1} \times U(1)_{2}$ symmetry. Under $U(1)_{1} \times U(1)_{2}$, the quantum numbers of the fields are $N_{1} \equiv(1,0), N_{2} \equiv(0,1)$, $\Phi_{1} \equiv(2,0)$ and $\Phi_{2} \equiv(0,2)$ respectively. When the $\Phi_{i}(x)$ acquire VEV's, these global symmetries are broken and there will be two Nambu-Goldstone bosons.

We assume that the fields $\Phi_{i}$ acquire VEV's, and it is then useful to parametrize them as nonlinear $\sigma$-model fields in terms of the NGB's, $\phi_{i}$ and decay constants, $f_{i}$,

$$
\left\langle\Phi_{i}\right\rangle=\left(f_{i} / 2 \sqrt{2}\right) \exp \left(2 i \phi_{i} / f_{i}\right)
$$

and we have $\alpha_{i} \Phi_{i} \rightarrow M_{i} e^{2 i \phi_{i} / f_{i}}$. Here we assume a common value for the decay constants $f_{i}=f \sim M_{i}$. The $\phi_{i}$ are massless NGB's at this stage.

The $U(1)_{1} \times U(1)_{2}$ symmetry will generally be explicitly broken by the Yukawa interactions of the right-handed neutrinos with the left-handed leptons $\left(\ell_{i} \equiv\left\{\nu_{i}, e_{i}^{-}\right\}\right)$through the usual standard model Higgs doublet $\left(H_{0}\right)$. If we allow such symmetry breaking terms, there will be divergent contributions to the mass of the Nambu-Goldstone bosons. Only when the original symmetry is broken by soft terms, which may originate from electroweak symmetry breaking, then the mass of the pNGB remains finite and small. For this reason we introduce two new Higgs doublets $H_{1}$ and $H_{2}$, transforming under the $U(1)_{1} \times U(1)_{2}$ as $(+1,-1)$ and $(-1,+1)$ respectively, to make the theory invariant under $U(1)_{1} \times U(1)_{2}$. As- 
signing the $U(1)_{1} \times U(1)_{2}$ quantum numbers $(1,0)$ for $\ell_{1} \equiv\left(\begin{array}{c}\nu_{e} \\ e^{-}\end{array}\right)$and $(0,1)$ for $\ell_{2} \equiv\left(\begin{array}{c}\nu_{\mu} \\ \mu^{-}\end{array}\right)$ the $U(1)_{1} \times U(1)_{2}$ invariant Yukawa interactions are given by

$$
\mathcal{L}_{\text {mass }}=f_{11} \bar{N}_{1} \ell_{1} H_{0}+f_{12} \bar{N}_{1} \ell_{2} H_{1}+f_{21} \bar{N}_{2} \ell_{1} H_{2}+f_{22} \bar{N}_{2} \ell_{2} H_{0}
$$

The soft terms may also originate from some new physics at higher energies. The Higgs scalars $H_{1}$ and $H_{2}$ are candidates for cold dark matter of the universe, since they cannot decay into quarks or light leptons [14].

Once the Higgs fields develop electroweak scale VEVs these become Dirac mass terms. The complete neutrino mass matrix is then:

$$
\begin{aligned}
-\mathcal{L}_{\text {mass }}= & \frac{1}{2} M_{1} \bar{N}_{1} N_{1}^{c} e^{2 i \phi_{1} / f}+\frac{1}{2} M_{2} \bar{N}_{2} N_{2}^{c} e^{2 i \phi_{2} / f}+m e^{i \alpha} \bar{N}_{1} \nu_{1}+m \epsilon e^{i \beta} \bar{N}_{1} \nu_{2} \\
& +\lambda m \epsilon^{\prime} e^{i \gamma} \bar{N}_{2} \nu_{1}+\lambda m e^{i \xi} \bar{N}_{2} \nu_{2} .
\end{aligned}
$$

We've introduced an overall Dirac mass parameter $m$ and scaling parameters $\lambda, \epsilon, \epsilon^{\prime}$, and we've also included all the phases which contribute to $\mathrm{CP}$ violation.

A three Higgs doublet model like the one presented above could also have interesting collider phenomenology, as it has a much richer electroweak symmetry breaking structure than the Standard Model. For our purposes it is sufficient to have the three scalars develop an electroweak scale vev which would lead to the Dirac masses above.

Note that we can make phase redefinitions on the neutrino fields $N_{i}$ and $\nu_{i}$ to try to absorb the majoron fields out of the mass matrix. Transforming the neutrinos along with the redefinition of the $\mathrm{CP}$ phases, we can bring the mass matrix into the form:

$$
\begin{aligned}
-\mathcal{L}_{\mu}= & \frac{1}{2} M_{1} \bar{N}_{1} N_{1}^{c} e^{2 i \phi / f}+\frac{1}{2} M_{2} \bar{N}_{2} N_{2}^{c}+m \bar{N}_{1} \nu_{1}+m \epsilon e^{i \eta} \bar{N}_{1} \nu_{2} \\
& +\lambda m \epsilon^{\prime} e^{i \eta} \bar{N}_{2} \nu_{1}+\lambda m \bar{N}_{2} \nu_{2}+\text { h.c. }
\end{aligned}
$$

where $2 \eta=\gamma-\alpha+\beta-\xi$ and $\phi=\phi_{1}-\phi_{2}$. It is not possible by further transformations to remove the field $\phi$ or phase $\eta$ from the mass terms. The combination $\phi_{1}+\phi_{2}$ has been absorbed out of the mass terms and remains a massless NGB, while the field $\phi=\phi_{1}-\phi_{2}$ 
becomes a pNGB, due to the explicit breaking of the $U(1)_{1-2}$ symmetry by the Higgs-Yukawa terms.

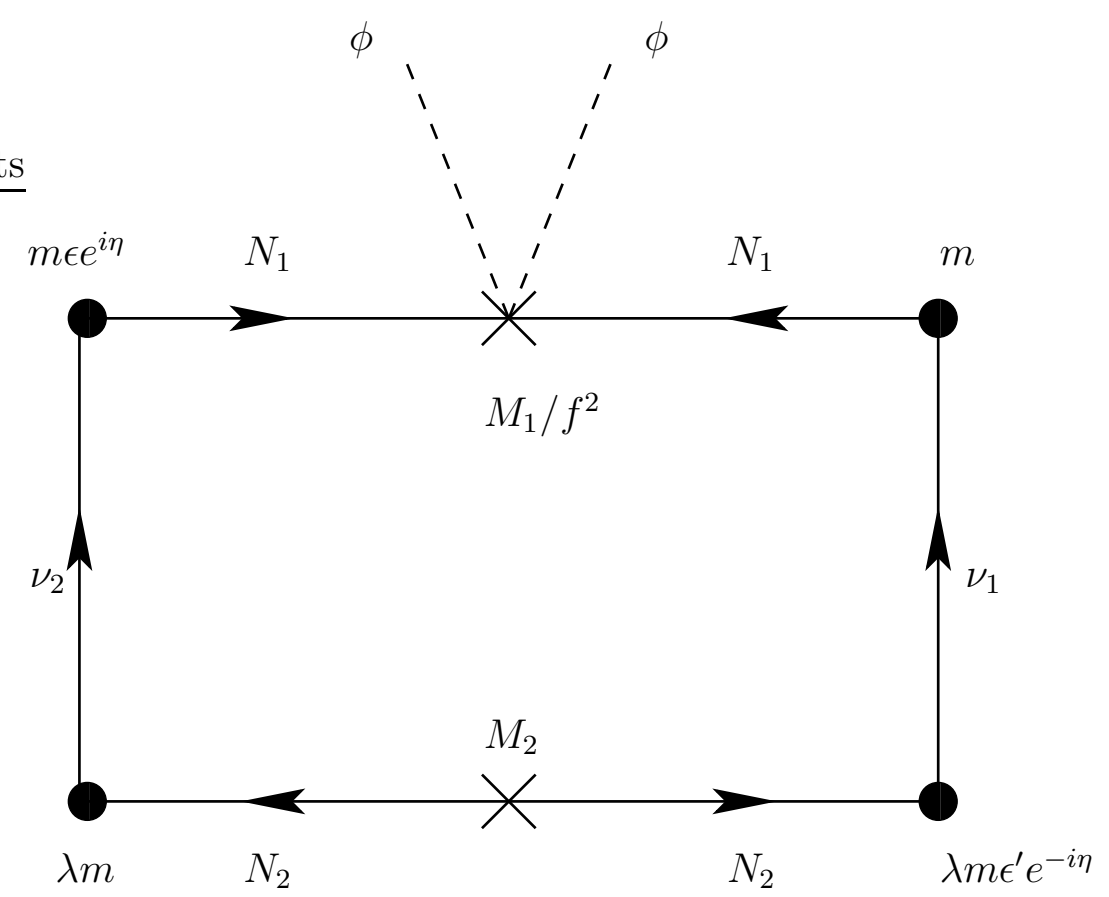

Figure 1: Loop diagram for the effective potential

The interaction of the scalar field through the Majorana and Dirac terms generates a Colemann-Weinberg effective potential for $\phi$. This can be estimated through the leading loop in Fig. 1. It has the remarkable property that the symmetry structure of the theory makes the loop finite. The reason is that the $\phi$ field could be eliminated if any of the vertices is set to zero. The vertices in this diagram come after electroweak symmetry breaking. Before the electroweak symmetry breaking the Nambu-Goldstone boson corresponding to $U(1)_{1-2}$ remains massless. After the electroweak symmetry breaking these vertices appear as soft terms and hence they cannot introduce non-renormalizable terms that need to be counter balanced.

Note that the phase $\eta$ disappears from the potential and the field $\phi$ cannot be removed from the diagram by rephasing of any fields. More generally, the diagram is invariant under 
any field rephasings involving $\phi_{i}$ or other CP phases.

An explicit calculation gives:

$$
V_{e f f}\left(\phi^{2}\right)=-\frac{m^{4} \lambda^{2} \epsilon \epsilon^{\prime}}{2 \pi^{2}} \frac{M_{1} M_{2} \log \left(\frac{M_{1}^{2}}{M_{2}^{2}}\right)}{M_{1}^{2}-M_{2}^{2}} \cos \left(\frac{2 \phi}{f}\right)
$$

The potential for the two generation model depends on all coupling constants, is weakly dependent on the heavy scales $M_{i}$, and has minima at $\phi=0, \pi f, 2 \pi f, \cdots$. We can expand the potential around one minimum obtaining a constant term, a mass term and higher orders in $\phi$ giving interactions:

$$
V_{e f f}(\phi)=\frac{m^{4} \lambda^{2} \epsilon \epsilon^{\prime}}{2 \pi^{2}} \frac{M_{1} M_{2} \log \left(\frac{M_{1}^{2}}{M_{2}^{2}}\right)}{M_{1}^{2}-M_{2}^{2}}\left(-1+2 \frac{\phi^{2}}{f^{2}}-O\left(\frac{\phi^{4}}{f^{4}}\right)+\cdots\right) .
$$

Thus the induced mass of the field $\phi$ is now:

$$
m_{\phi}=\frac{m^{2} \lambda \sqrt{\epsilon \epsilon^{\prime}}}{\pi f} \frac{M_{1} M_{2} \log \left(\frac{M_{1}^{2}}{M_{2}^{2}}\right)}{M_{1}^{2}-M_{2}^{2}} .
$$

If $M_{1}=M_{2}$ all the $M_{i}$ dependent factor in the equation above becomes 1. As mentioned earlier, the symmetry at the scale $f \sim M_{i}$ protects the mass of the pseudo Nambu-Goldstone boson, so an explicit breaking of the symmetry at the scale $m$ can generate a mass of the order of $m^{2} / f$. Thus the mass of the scalar is very small. We now turn to the see-saw mechanism, long-range force, leptogenesis and the origin of dark energy in this model.

\section{Neutrino Masses and Long Range Forces Among Neu- trinos}

We shall next study the neutrino masses in this scenario. If we ignore the small mass varying effect on the neutrino masses coming from the pNGB, the time-development of the light states is determined by the matrix

$$
\left.-\mathcal{L}_{e f f}=\overline{\nu^{c}} m_{D}^{T} M_{R}^{-1} m_{D} \nu=\frac{m^{2}}{M}\left[\begin{array}{ll}
\overline{\nu_{1}^{c}} & \overline{\nu_{2}^{c}}
\end{array}\right)\left(\begin{array}{cc}
1+\left(\lambda \epsilon^{\prime}\right)^{2} e^{2 i \eta} & e^{i \eta}\left(\epsilon+\lambda^{2} \epsilon^{\prime}\right) \\
e^{i \eta}\left(\epsilon+\lambda^{2} \epsilon^{\prime}\right) & \lambda^{2}+\epsilon^{2} e^{2 i \eta}
\end{array}\right)\left(\begin{array}{l}
\nu_{1} \\
\nu_{2}
\end{array}\right)\right]
$$


where we have assumed $M_{1} \simeq M_{2}=M$. This mass matrix can be diagonalized to

$$
m^{\text {diag }}=\left(\begin{array}{cc}
m_{1} e^{i \theta_{1}} & 0 \\
0 & m_{2} e^{i \theta_{2}}
\end{array}\right) .
$$

The mixing angle is:

$$
\tan 2 \theta=-\frac{2\left(\epsilon+\epsilon^{\prime} \lambda^{2}\right) \sqrt{\left(1+\epsilon^{2}\right)^{2}+\left(1+\epsilon^{\prime 2}\right)^{2} \lambda^{4}+2\left(1+\epsilon^{2}\right)\left(1+\epsilon^{\prime 2}\right) \lambda^{2} \cos 2 \eta}}{1-\epsilon^{4}-\lambda^{4}\left(1-\epsilon^{\prime 4}\right)-2\left(\epsilon^{2}-\epsilon^{\prime 2}\right) \lambda^{2} \cos 2 \eta} .
$$

We would like to reproduce the maximal mixing observed for atmospheric neutrinos. One possibility that immediately gives this result is to have $\epsilon, \epsilon^{\prime} \ll 1$ and $\lambda \approx 1$, up to terms of order $\epsilon_{i}^{2}$. Keeping only the leading terms in $\epsilon, \epsilon^{\prime}$ or $1-\lambda$, the mixing angle becomes:

$$
\tan 2 \theta=\frac{2\left(\epsilon+\epsilon^{\prime}\right) \cos \eta}{-2(1-\lambda)+\left(\epsilon^{2}-\epsilon^{\prime 2}\right) \cos 2 \eta} .
$$

The neutrino masses are given by:

$$
m_{1} \simeq \frac{m^{2}}{M}\left[1+\left(\epsilon+\epsilon^{\prime}\right) \cos \eta\right]
$$

and

$$
m_{2} \simeq \frac{m^{2}}{M}\left[1-\left(\epsilon+\epsilon^{\prime}\right) \cos \eta\right]
$$

The mass squared difference, which should be in the atmospheric range of $\sim 2 \times 10^{-3} \mathrm{eV}^{2}$, is of order $\epsilon_{i}: \Delta m^{2}=-4 m^{4}\left(\epsilon+\epsilon^{\prime}\right) \cos \eta / M^{2}$. Since the $\epsilon_{i}$ parameters were assumed to be small, the required right-handed neutrino mass scale should be lower than in standard seesaw type scenarios.

As we go a step further and keep the linear term in the scalar field, we obtain in addition an interaction between the neutrinos and scalar field. The exchange of the scalar particle between the eigenstates results in a new force whose range is at our disposal. We are free to vary the mass of the scalar by selecting values for the decay constant $f$. The final mass and interaction Lagrangian, up to terms linear in $\epsilon, \epsilon^{\prime}$, is given by

$$
\mathcal{L}=\frac{m^{2}}{2 M} \overline{\Psi^{c}}\left\{\left(\begin{array}{cc}
m_{1} & 0 \\
0 & m_{2}
\end{array}\right)-i \frac{\phi}{f}\left(\begin{array}{cc}
e^{i \alpha_{1}} & -e^{i \alpha_{2}} \\
-e^{i \alpha_{2}} & e^{i \alpha_{1}}
\end{array}\right)\right\} \Psi+\text { H.C. }
$$

The off-diagonal long range force predicted by the couplings of the pNGB in this model could have direct consequences in neutrino oscillation experiments [18] or in cosmology [1, 2]. 

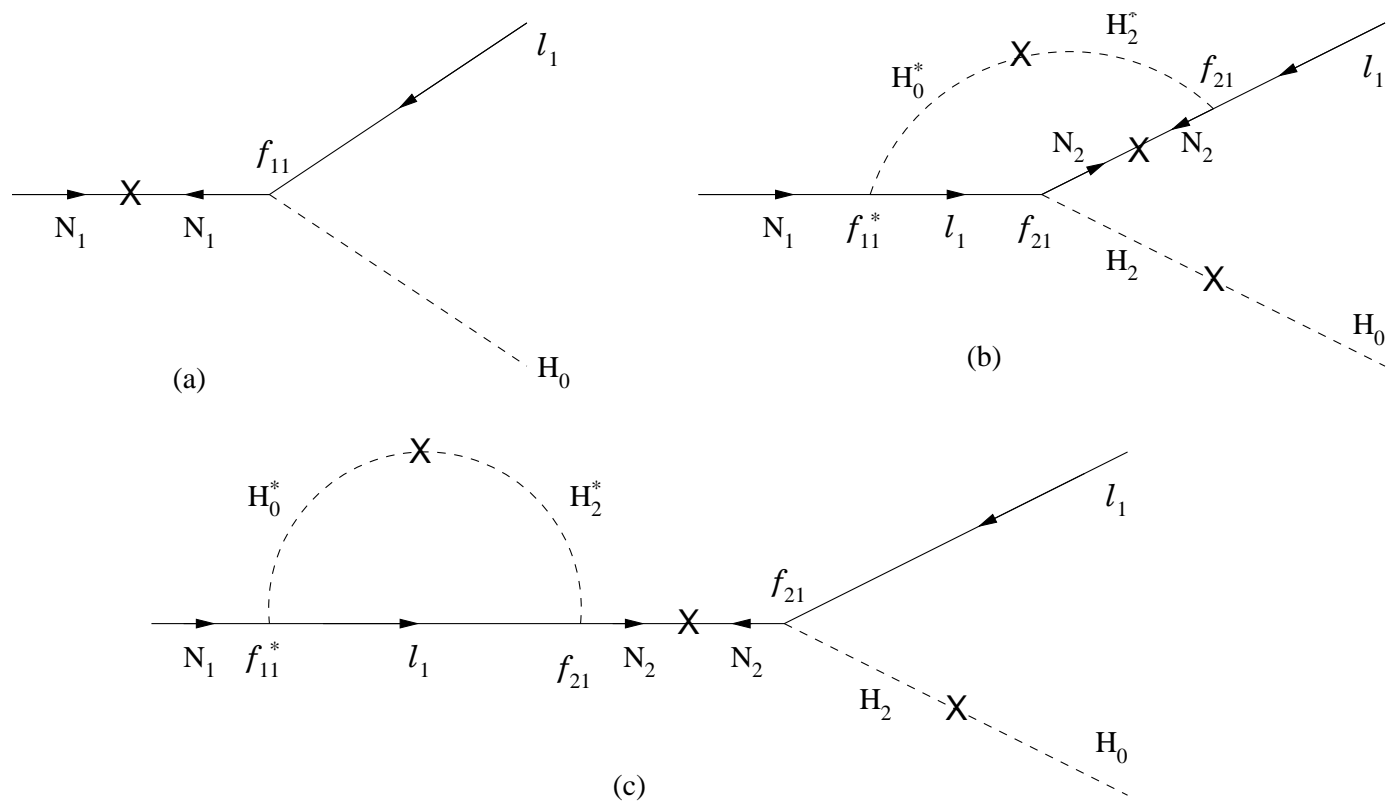

Figure 2: Tree level and one-loop diagrams that contributes to the generation of lepton asymmetry by the decays of $N_{1}$.

\section{Lepton Asymmetry of the Universe}

The decays of the right-handed neutrinos

$$
\begin{aligned}
N_{i} & \rightarrow \ell_{j}+H_{a}^{\dagger} \\
& \rightarrow \ell_{j}^{c}+H_{a}
\end{aligned}
$$

violate lepton number and $\mathrm{CP}$ violation comes from the $\mathrm{CP}$ phase $\eta$. There are vertex-type [15, 16] and self-energy [17] one-loop diagrams that interfere with the tree-level decays to give an asymmetry as shown in figure 2, The generation of a baryon asymmetry requires one more important ingredient, namely, the mixing of the Higgs doublets $H_{0,1,2}$. The quartic couplings $\left|H_{0}\right|^{2}\left|H_{i}\right|^{2}$ give all the required couplings which are not suppressed. We denote the mixing of $H_{0}$ with $H_{i}$ by $V_{0 i}$.

We assume that $N_{2}$ is heavier than $N_{1}\left(M_{1}<M_{2}\right)$ so that $N_{2}$ decays first and at a later 
time the decays of $N_{1}$ contribute to the asymmetry. The interference of the tree-level and one-loop diagrams in figure 2 generate the asymmetry. The cross on a fermion line indicates a Majorana mass insertion and the cross on a Higgs line refers to the mixing between the scalars. The tree-level and one-loop diagrams that contribute to the generation of lepton asymmetry in the decays of $N_{1}$ are shown in figure 2. There will be similar diagrams contributing to the process $N_{1} \rightarrow \ell_{2}+H_{1}$.

The interference between the diagrams give the asymmetry

$$
\begin{aligned}
\delta & =\frac{\Gamma\left(N_{1} \rightarrow \ell H^{\dagger}\right)-\Gamma\left(N_{1} \rightarrow \ell^{c} H\right)}{\Gamma\left(N_{1} \rightarrow \ell H^{\dagger}\right)+\Gamma\left(N_{1} \rightarrow \ell^{c} H\right)} \\
& =\frac{3}{16 \pi} \frac{M_{1}}{M_{2}} \frac{m^{2} \lambda^{2}}{v_{1}^{2}+v_{0}^{2} \epsilon^{2}}\left[\left|V_{02}\right|^{2} \frac{v_{1}^{2}}{v_{2}^{2}} \epsilon^{\prime 2}-\left|V_{01}\right|^{2} \epsilon^{2}\right] \sin 2 \eta .
\end{aligned}
$$

where $v_{a}=\left\langle H_{a}\right\rangle, a=0,1,2$. One interesting feature of this model is that the CP violating phase $\eta$ is solely responsible for the generation of the lepton asymmetry of the universe and the sign of the asymmetry is also determined in terms of the parameters of this model. This phase may also be observed in lepton number violating processes like neutrinoless double beta decay.

At temperatures above $T>10^{9} \mathrm{GeV}$, the out-of-equilibrium condition can be satisfied

$$
\Gamma_{N_{1}}=\frac{\left|f_{1 i}\right|^{2}}{16 \pi} M_{1}<H\left(M_{1}\right)=1.7 \sqrt{g_{*}} \frac{T^{2}}{M_{P l}} \quad \text { at } T=M_{1} .
$$

However, for thermal production of the right-handed neutrinos, the interaction rate $\left(\Gamma_{N_{1}}\right)$ should not be much smaller than the expansion rate of the universe $(H)$. Considering the requirement of thermal production and subsequent wash out of the asymmetry, in addition to the suppression factor $\kappa=\Gamma_{N_{1}} / H\left(M_{1}\right)$, which enters if the decay rate is slightly greater than the expansion rate of the universe, the amount of $(B-L)$ asymmetry is given by

$$
\frac{n_{B-L}}{s} \approx-\frac{40}{g_{*} \pi^{4}} \frac{\delta}{\kappa}
$$

The sphaleron interactions now convert this $(B-L)$ asymmetry to a baryon asymmetry

$$
\frac{n_{B}}{s}=\frac{24+4 n_{H}}{66+13 n_{H}} \frac{n_{B-L}}{s}
$$


where $n_{H}$ is the number of Higgs doublets in the standard model. This can then generate the required amount of baryon asymmetry of the universe.

\section{Origin of Dark Energy}

The basic idea behind the neutrino dark energy $(\nu D E)$ models is that the neutrino mass varies as a function of a light scalar field $\mathcal{A}$ (the acceleron) [11] and the mass of the acceleron today should be less than $\sim\left(10^{-4} \mathrm{eV}\right)$. In the present model, the pNGB $\mathcal{A}=(i \phi)$ corresponding to the soft $U(1)_{A-B}$ global symmetry breaking takes the role of the acceleron $(\mathcal{A})$. Thus the mass matrix becomes

$$
\begin{aligned}
\mathcal{L}_{\mu}= & \frac{1}{2} M_{1}(\mathcal{A}) \bar{N}_{1}^{c} N_{1}+\frac{1}{2} M_{2} \bar{N}_{2}^{c} N_{2}+m \bar{N}_{1} \nu_{1}+m \epsilon \bar{N}_{1} \nu_{2} \\
& +\lambda m \epsilon^{\prime} \bar{N}_{2} \nu_{1}+\lambda m \bar{N}_{2} \nu_{2}+\text { h.c. }
\end{aligned}
$$

where $M_{1}(\mathcal{A})$ is the mass of the heavy right-handed neutrino $N_{1}$ that varies explicitly with the acceleron field $\mathcal{A}$. It is interesting to note that $M_{1}(\mathcal{A})$ is specified and the effective neutrino mass also varies explicitly $m_{\nu}(\mathcal{A})=m^{T} M^{-1}(\mathcal{A}) m$, as required by some models of mass varying neutrinos. The present model has the same generic problems like any other models of mass varying neutrinos [20], some of which have been taken care of in variants of this model [21], but the main feature of the present model is that it explains the origin of the acceleron field as the pseudo Nambu-Goldstone boson (pNGB).

It is also possible to construct scenarios where the pseudo Nambu-Goldstone boson acts as cold dark matter [19] or can lead to non-standard structure-formation due to the strong long range interaction among neutrinos.

\section{Summary}

A large Majorana scale for neutrino masses is a necessary component of the see-saw mechanism. At this very high energy scale there could be global symmetries which are broken in 
order to produce Majorana masses. Remnants of the symmetries may exist at low energy. In this article we proposed a generic model with global symmetry. The breaking of this global symmetry gives masses to right-handed neutrinos and produces Nambu-Goldstone bosons.

In this framework we investigated a model with two generations of neutrinos and scalar particles. We showed that the Dirac masses of the neutrinos appearing from the electroweak symmetry breaking implies soft breaking of this global symmetry, which generates an effective potential that is finite. The Nambu-Goldstone boson then acquires a mass and produces a long-range force between neutrinos. Their masses have an explicit dependence on the scalar field, which may bring additional density effects producing cosmological consequences.

The model has other attractive properties. The decays of the heavy neutrinos generate a lepton asymmetry consistent with the scenario of leptogenesis. It also describes the masses and mixings of light neutrinos. The pNGB can play the role of the "acceleron" field introduced in models of mass varying neutrinos.

\section{Acknowledgement}

C.T. Hill thanks Dortmund University for the award of a Gambrinus Fellowship and its hospitality during the early stages of this work. E.A. Paschos thanks the Theory Group of Fermilab for its hospitality. U. Sarkar thanks the Institut für Physik, Univ. Dortmund and Alexander von Humboldt Foundation for their support. The work of I. Mocioiu was supported in part by the National Science Foundation grant PHY-0555368 adn the work for two of us (EAP and US) by BMBF, Bonn under contract 05 HT 4PEA/9. 


\section{References}

[1] C.T. Hill, D.N. Schramm and J.N. Fry, Nucl. Part. Phys. 19, 25 (1989)

[2] J. A. Frieman, C. T. Hill and R. Watkins, Phys. Rev. D 46, 1226 (1992); A. K. Gupta, C. T. Hill, R. Holman and E. W. Kolb, Phys. Rev. D 45, 441 (1992); J. A. Frieman, C. T. Hill, A. Stebbins and I. Waga, Phys. Rev. Lett. 75, 2077 (1995) arXiv:astro-ph/9505060].

[3] M. Gell-Mann, P. Ramond and R. Slansky, Rev. Mod. Phys. 50, 721 (1978); M. GellMann, P. Ramond, and R. Slansky, in Supergravity, edited by P. van Nieuwenhuizen and D. Z. Freedman (North-Holland, Amsterdam, 1979), p. 315; T. Yanagida, in Proceedings of the Workshop on the Unified Theory and the Baryon Number in the Universe, edited by O. Sawada and A. Sugamoto (KEK Report No. 79-18, Tsukuba, Japan, 1979), p. 95; P. Minkowski, Phys. Lett. B 67, 421 (1977); R. N. Mohapatra and G. Senjanovic, Phys. Rev. Lett. 44, 912 (1980); J. Schechter and J.W.F. Valle, Phys. Rev. D 22, 2227 (1980); Phys. Rev. D 25, 774 (1982).

[4] G. B. Gelmini and M. Roncadelli, Phys. Lett. B 99, 411 (1981). Y. Chikashige, R. N. Mohapatra and R. D. Peccei, Phys. Lett. B 98, 265 (1981).

[5] R.D. Peccei and H.R. Quinn, Phys. Rev. Lett. 38, 1440 (1977); Phys. Rev. D 16, 1791 (1977); S. Weinberg, Phys. Rev. Lett. 40, 223 (1978); F. Wilczek, Phys. Rev. Lett. 40, 279 (1978).

[6] H. Georgi and A. Pais, Phys. Rev. D 10, 539 (1974).

[7] C.T. Hill and G.G. Ross, Phys. Lett. B 203, 125 (1988); Nucl. Phys. B 311, 253 (1998).

[8] N. Arkani-Hamed, A. G. Cohen and H. Georgi, Phys. Rev. Lett. 86, 4757 (2001); C. T. Hill, S. Pokorski and J. Wang, Phys. Rev. D 64, 105005 (2001). 
[9] C. T. Hill and A. K. Leibovich, Phys. Rev. D 66, 075010 (2002) arXiv:hep-ph/0205237.

C. T. Hill and A. K. Leibovich, Phys. Rev. D 66, 016006 (2002) arXiv:hep-ph/0205057.

[10] C. Wetterich, Nucl. Phys. B302, 668 (1988); P. J. E. Peebles and B. Ratra, Astrophys. J. 325, L17 (1988).

[11] R. Fardon, A. E. Nelson, and N. Weiner, JCAP 0410, 005 (2004); see also P. Q. Hung, hep-ph/0010126; P. Gu, X. Wang, and X. Zhang, Phys. Rev. D68, 087301 (2003).

[12] D. B. Kaplan, A. E. Nelson, and N. Weiner, Phys. Rev. Lett. 93, 091801 (2004); G. Dvali, Nature 432, 567 (2004).

[13] X.-J. Bi, P. Gu, X. Wang, and X. Zhang, Phys. Rev. D69, 113007 (2004); P.-H. Gu and X.-J. Bi, Phys. Rev. D70, 063511 (2004).

[14] R. Barbieri, L.J. Hall and V.S. Rychkov, Phys. Rev. D 74 (2006) 015007; E. Ma, Mod. Phys. Lett. A 21 (2006) 1777.

[15] M. Fukugita and T. Yanagida, Phys. Lett. B174, 45 (1986).

[16] M.A. Luty, Phys. Rev. D45, 455 (1992); W. Buchmuller, R. Peccei and T. Yanagida, Ann. Rev. Nucl. Part. Sci. 55, 311 (2005).

[17] M. Flanz, E.A. Paschos and U. Sarkar, Phys. Lett. B 345, 248 (1995); M. Flanz, E.A. Paschos, U. Sarkar and J. Weiss, Phys. Lett. B 389, 693 (1996).

[18] A. S. Joshipura and S. Mohanty, Phys. Lett. B 584, 103 (2004); J. A. Grifols and E. Masso, Phys. Lett. B 579, 123 (2004); V. Barger, P. Huber, and D. Marfatia, Phys. Rev. Lett. 95, 211802 (2005); M. Cirelli, M. C. Gonzalez-Garcia, and C. Pena-Garay, Nucl. Phys. B719, 219 (2005); M. C. Gonzalez-Garcia, P. C. de Holanda, E. Masso and R. Zukanovich Funchal, arXiv:hep-ph/0609094; A. Bandyopadhyay, A. Dighe and A. S. Joshipura, arXiv:hep-ph/0610263; A. Bandyopadhyay, A. Dighe and A. S. Joshipura, arXiv:hep-ph/0611038. 
[19] S. Das and N. Weiner, arXiv:astro-ph/0611353.

[20] R. D. Peccei, Phys. Rev. D71, 023527 (2005); N. Afshordi, M. Zaldarriaga, K. Kohri, Phys. Rev. D72, 065024 (2005); X.-J. Bi, B. Feng, H. Li, and X. Zhang, Phys. Rev. D72, 123523 (2005); A. W. Brookfield, C. van de Bruck, D. F. Mota, and D. TocchiniValentini, Phys. Rev. Lett. 96, 061301 (2006), astro-ph/0512367; H. Li, B. Feng, J.-Q. Xia, and X. Zhang, Phys. Rev. D 73, 103503 (2006).

[21] R. Barbieri, L. J. Hall, S. J. Oliver, and A. Strumia, Phys. Lett. B625, 189 (2005); R. Takahashi and M. Tanimoto, Phys. Lett. B633, 675 (2006); R. Fardon, A. E. Nelson, and N. Weiner, JHEP 0603, 042 (2006); E. Ma and U. Sarkar, Phys. Lett. B 638, 358 (2006). 